\title{
Multidimensional Poverty Modeling for Namibia Using the Beta Distribution
}

\author{
Ndubano Mafale ${ }^{1}$, Dismas Ntirampeba ${ }^{1}$, Jacob Ong'ala ${ }^{1}$ \\ ${ }^{1}$ Department of Mathematics and Statistics, Namibia University of Science and Technology, Windhoek, Namibia \\ Correspondence: Ndubano Mafale, Department of Mathematics and Statistics, Namibia University of Science and \\ Technology, Windhoek, Namibia. Tel: +264 81807 4857. E-mail: ndubanom@gmail.com
}

Received: September 27, 2021

Accepted: October 27, $2021 \quad$ Online Published: October 28, 2021

doi:10.5539/ijsp.v10n6p47

URL: https://doi.org/10.5539/ijsp.v10n6p47

\begin{abstract}
Despite global efforts in alleviating poverty, many people are still living in poverty. Different methods were employed to estimate poverty with many researchers moving from monetary to multidimensional poverty modeling approach. In Namibia, very few studies have been conducted to estimate poverty in a multidimensional sense. The 2015/2016 Namibia household income and expenditure survey dataset was employed to develop multidimensional poverty indices (MPIs) using beta distribution. We showed that the MPI is equivalent to the mean of the left truncated beta distribution. The results revealed that the northern regions of Namibia are the most affected by multidimensional poverty. The results from this study can be used to identify areas that are severely affected by poverty and consequently form a basis to develop appropriate measures intended to alleviate poverty.
\end{abstract}

Keywords: entropy weight, left truncated beta distribution, multidimensional poverty index

\section{Introduction}

Poverty is a global phenomenon that negatively affects human kind especially in developing countries. Despite numerous efforts to alleviate it, poverty remains an unresolved issue in many countries (Nam, 2020). Some studies view poverty as an income deprivation (Bellù \& Liberati, 2005; Jolliffe \& Prydz, 2016). Others argued that understanding poverty as only a lack of financial resources undermines and ignores other deprivations that people may be experiencing, such as health, education, and social needs, that are not limited to economic resources but go beyond (Mothkoor \& Badgaiyan, 2021). Poverty can therefore be understood beyond one-dimensional setup by considering deprivations in different domains i.e. multidimensional setup (Santos \& Alkire, 2011).

Traditionally, poverty has been measured based on the one-dimensional approach which is usually income or consumption expenditure (Pochun, 2002; Santos \& Alkire 2011). In this approach, a container of goods and services regarded as the minimum requirement needed for an individual or household to live a non-poor life is estimated at the market prices, and individuals or households who do not meet the minimum requirement are deemed poor (Santos \& Alkire, 2011). One-dimensional poverty, also referred to as income poverty, uses poverty lines to categorize if a person is considered poor or not. These lines are useful in monitoring the progress made in alleviating poverty as they can be used to depict the percentage of individuals below the poverty line (Pochun, 2002).

Income poverty can be classified as absolute or relative (Decerf, 2017). According to De (2017), absolute poverty is defined as a shortage of a person's basic needs and services, which are mostly linked to food, clothes, and housing. Absolute poverty lines consider the poverty status of each individual or household independent of the welfare situations of other individuals or households belonging to the same society (Bellù \& Liberati, 2005). More specifically, they set up a minimum amount of money needed to cover some expenses such as rent or food. On the other hand, relative poverty is defined as an individual's failure to reach a certain level of their community's median income (typically in the range of 40-60\% of the median income) (Mellish, 2016). This approach is based on relative poverty lines with an income threshold that is dependent on the income standard. An individual is considered poor if his/her income falls below the standard income of his community (Decerf, 2017). This approach of poverty is regarded as a measure of inequality as individuals are considered poor if they are economically or socially disadvantaged relative to other people in their community (De, 2017).

Mellish (2016) argued that relative poverty does not take into account the social and economic changes that take place in a community. He further claimed that the comparison of poverty between communities or the same community at 
different times is impossible as communities may have different levels of relative poverty compared to other communities and consequently the relative poverty is deemed not to be a good measure of poverty.

Allen (2017) proposed a new basis for poverty measurement based on linear programming for identifying the least expensive diet and clear budgeting for non-food spending. The results showed that this approach is far better than the dollar-a-day poverty line as it is explicitly related to survival and well-being, and it can be used to do comparisons across time. Although this approach is better in estimating poverty, it still ignores other humanistic needs of individuals such as education and health. Ive and Bosch (2007) pointed out that there is a need for a more credible and grounded poverty standard that can complement or possibly replace relative poverty. They argue that the current relative poverty lines exclude the possibility that absolute or primary poverty exists.

The one-dimensional (absolute and relative poverty lines) approach of measuring poverty using monetary indicators such as income and consumption expenditure leads to partial and limited understanding of poverty which results in ineffective poverty reduction interventions. Also, it underestimates poverty as aspects of deprivation such as education and health are not captured, as a result the devised interventions to eliminate poverty will only work in the short term (Appiah-Kubi, Amanning-Ampomah, \& Ahortor, 2007). Santos and Alkire (2011) criticized the income poverty approach in different ways. Firstly, income is not good at determining if people have access to goods and services that they find important in life as some key needs may not be met in the market. For example, access to education and health facilities, which are mostly provided by the government. Secondly, each household has a different way of turning income into necessary needs. Some individuals may not be able to access the container of goods and services that they should be able to access on paper with their income due to some disadvantages such as lack of education or being disabled. Finally, income is just a means to an end and what is more important is the ends, not the means. For example, when it comes to nutrition we are more interested in whether an individual is well-nourished, not whether they have the income to be well-nourished. All these loopholes in one-dimensional poverty show the need for a more inclusive approach that considers other aspects of poverty (Appiah-Kubi et al., 2007).

Recent studies on poverty show that it is being accepted that poverty is not just a lack of income or resources needed to meet basic needs but it is a multidimensional phenomenon that looks at poverty in more than one dimension (De, 2017; Walker, 2019; Appiah-Kubi et al., 2007; Ciani, Gagliardi, Riccarelli, \& Betti, 2019; Nam, 2020; Mothkoor \& Badgaiyan, 2021; Betti, D’Agostino, \& Neri, 2002; Walker, Tomlinson, \& Williams, 2008; Adepoju, 2018). A multidimensional approach to estimating poverty is practical, broad, more insightful because it measures poverty in multiple dimensions. A Multidimensional Poverty Index (MPI) is used to measure multidimensional poverty by capturing multiple deprivations that poor individuals experience. The MPI captures poverty in terms of health, education, living standards, and other indicators. The strength of this approach lies in its ability to not only capture poor individuals or households but also capturing how poor they are (Mothkoor \& Badgaiyan, 2021).

Thus, the MPI combines two important pieces of information: the incidence of poverty $(\mathrm{H})$ and the intensity of the deprivation the poor experience (A). The recent conceptualization of poverty as a multidimensional phenomenon has led researchers to study multidimensional poverty using different methods (Adepoju, 2018). Some of these methods include the Alkire-Foster method (Mukui, 2017), Markov model (Adepoju, 2018) latent class model (Nam, 2020), fuzzy theory (Appiah-Kubi et al., 2007), Ciani et al., 2019)), multinomial logistic regression (Adepoju, 2018), panel regression models (Betti et al., 2002), and structural equation modeling (Walker et al., 2008).

To the knowledge of the researchers, little has been done in Namibia to estimate poverty in a multidimensional sense. The only study on multidimensional poverty carried out in Namibia known to the researcher is the 2021 multidimensional poverty index for Namibia by the Namibia Statistics Agency [NSA] (2021). Another one that came close was the index of multiple deprivations by the National Planning Commission [NPC] (2015). Even though it measured deprivation in multiple dimensions, they modified the global recommended indicators without justification. This paper introduces a new method (beta distribution) of computing the multidimensional poverty index which can be used to assess poverty at national and regional levels, and identify the most affected areas for targeted interventions.

\section{Materials and Method}

\subsection{Type and Sources of Data}

This study used the 2015/2016 Namibia Household Income and Expenditure Survey (NHIES) secondary data collected by the Namibia Statistics Agency (NSA) in association with the Ministry of Economic Planning, the World Bank (WB), and the United States Census Bureau. The survey covered the country's 14 regions.

\subsection{Sampling Procedure and Sample size}

Households in the survey were chosen using the stratified two-stage cluster sampling method. In the first stage, geographical areas classified as primary sampling units (PSUs) were selected using the probability proportional to size 
(PPS) sampling jointly with a systematic sampling procedure from the national sampling frame (NSF). A total of 864 PSUs were selected in total using this procedure. The second stage units were the households. In this stage, 12 sample households per PSU were selected, resulting in 10368 households being sampled as shown in Table 1. However, only 10090 households with complete information were used in this study.

Table 1. Distribution of sample PSUs and households by region

\begin{tabular}{|c|c|c|c|c|}
\hline Region & PSU & $\begin{array}{c}\text { Sample } \\
\text { Households per } \\
\text { PSU }\end{array}$ & $\begin{array}{l}\text { Total sample } \\
\text { households }\end{array}$ & $\begin{array}{l}\text { Responding } \\
\text { households }\end{array}$ \\
\hline !Karas & 48 & 12 & 576 & 559 \\
\hline Erongo & 72 & 12 & 864 & 828 \\
\hline Hardap & 48 & 12 & 576 & 561 \\
\hline Kavango East & 48 & 12 & 576 & 554 \\
\hline Kavango West & 48 & 12 & 576 & 568 \\
\hline Khomas & 96 & 12 & 1152 & 1084 \\
\hline Kunene & 48 & 12 & 576 & 570 \\
\hline Ohangwena & 72 & 12 & 864 & 854 \\
\hline Omaheke & 48 & 12 & 576 & 557 \\
\hline Omusati & 72 & 12 & 864 & 854 \\
\hline Oshana & 72 & 12 & 864 & 846 \\
\hline Oshikoto & 72 & 12 & 864 & 852 \\
\hline Otjozondjupa & 72 & 12 & 864 & 837 \\
\hline Zambezi & 48 & 12 & 576 & 566 \\
\hline Total & 864 & & 10368 & 10090 \\
\hline
\end{tabular}

This study adopted the framework outlined by Santos and Alkire (2011) in which three domains (health, education, and living standard) are commonly used. The indicators for each of the domains used in this study are shown in Table 2.

Table 2. Indicators considered in this study

\begin{tabular}{|c|c|}
\hline Domain & Indicator \\
\hline Health & Nutrition (food adequacy in the past 7 days) \\
\hline Education & Years of schooling \\
\hline & School attendance \\
\hline Living standard & Cooking fuel \\
\hline & Sanitation \\
\hline & Drinking \\
\hline & Electricity \\
\hline
\end{tabular}

\subsection{Computation of Multidimensional Deprivation Scores}

Computing the deprivation scores requires the allocation of weight to each indicator. In this study, weights were derived using the entropy method (Gao \& Sun, 2020), although alternative weighting methods such as the equal weighting method (Santos \& Alkire, 2011), the CRITIC weighting method (Wang, Huang, Chen, Liu, \& Xue, 2018), the SMART weighting method (Oktavianti, Komala, \& Nugrahani, 2019), etc., may be considered. The deprivation scores were computed as a function of indicators as follows. Let $y_{i}$ be the deprivation score for each household, $I_{j}$ be the $j^{t h}$ indicator for $i^{\text {th }}$ household, and $W_{j}$ is the entropy weights of indicator $I_{j}$. Then, 


$$
y_{i}=\sum_{j=1}^{h} W_{j} I_{j}, i=1,2,3, \ldots \ldots \ldots, k \text { and } j=1,2,3 \ldots \ldots \ldots, h
$$

where $k$ is the total number of households and $h$ is the total number of indicators.

A household is considered to be deprived if $y_{i} \geq p$ (where $p \in(0,1)$ ). Otherwise, a household with a deprivation score below the threshold is considered non-deprived and its deprivation score is equated to zero. This whole process is referred to as censoring (Santos \& Alkire, 2011). We denote the censored deprivation scores by $y_{i}(p)$. That is,

$$
y_{i}(p)=\left\{\begin{array}{l}
y_{i} \text { if } y_{i} \geq p \\
0 \text { otherwise }
\end{array}\right.
$$

\subsection{Computation of Multidimensional Poverty Index (MPI)}

The computed deprivation scores are used to compute MPI, which combines two important components namely, the incidence of individuals who experience multiple deprivations and the intensity of the deprivation they experience (Santos \& Alkire, 2011). The incidence, also referred to as the headcount ratio is denoted by H, is computed as follows

$$
H=\frac{q}{n}
$$

where $q$ is the number of multidimensionally poor individuals, and $n$ is the total population. The intensity is referred to as the breadth of poverty and is denoted by A. It is computed using

$$
A=\frac{\sum_{i=1}^{k} y_{i}(p)}{q},
$$

Therefore, MPI is given as the product of Equation (3) and Equation (4), i.e.;

\subsection{Left Truncated Beta Distribution}

$$
M P I=H \times A
$$

Let $Y$ be a random variable with $y_{i}$ values in the interval $[a, b]$, and if the value of $\left(y_{i}\right)$ is less than $\mathrm{p}$ (the threshold value) we equate the values of $\left(y_{i}\right)$ to zeros. Then the probability density function of $Y$ is a beta distribution function given as;

$$
f_{a b}\left(y_{i} ; a, b, \alpha, \beta\right)=\frac{(b-a)\left(y_{i}-a\right)^{\alpha-1}\left(b-y_{i}\right)^{\beta-1}}{b^{\alpha+\beta-1} b\left(\frac{b-a}{b}\right)^{\alpha+\beta} B(\alpha, \beta)} ; a<y_{i}<b,
$$

where $\alpha$ and $\beta$ are the shape parameters, $a$ and $b$ are the lower and upper bounds, respectively (Zaninetti, 2013). Its mean is

$$
E(Y ; a, b, \alpha, \beta)_{a b}=\frac{\alpha b+a \beta}{\alpha+\beta}
$$

and its variance is given by

$$
\sigma^{2}(Y ; a, b, \alpha, \beta)_{a b}=\frac{(a-b)^{2} \alpha \beta}{(\alpha+\beta+1)(\alpha+\beta)^{2}}
$$

A left truncated beta distribution can be obtained from Equation (6) by replacing $a$ with any real number greater than $a$ but less than $b$. For this paper, $y_{i}(p) \in[p, 1]$ and Equation (6) becomes,

$$
\begin{aligned}
f\left(y_{i}(p) ; p, 1, \alpha, \beta\right) & =\frac{(1-p)\left(y_{i}(p)-p\right)^{\alpha-1}\left(1-y_{i}(p)\right)^{\beta-1}}{(1-p)^{\alpha+\beta} B(\alpha, \beta)} \\
& =\frac{1}{B(\alpha, \beta)} \frac{\left(y_{i}(p)-p\right)^{\alpha-1}\left(1-y_{i}(p)\right)^{\beta-1}}{(1-p)^{\alpha+\beta-1}} \\
& =\frac{\Gamma(\alpha+\beta)}{\Gamma(\alpha) \Gamma(\beta)} \frac{\left(y_{i}(p)-p\right)^{\alpha-1}\left(1-y_{i}(p)\right)^{\beta-1}}{(1-p)^{\alpha+\beta-1}} ; p \leq y_{i}(p)<1
\end{aligned}
$$

The expected mean of the left truncated beta distribution can be derived from Equation (9) as follows;

$$
E(Y)=\int_{p}^{1} y_{i}(p) f\left(y_{i}(p)\right) d y_{i}(p)
$$

We start by rewriting Equation (9) as follows; 


$$
\begin{gathered}
f\left(y_{i}(p)\right)=\frac{1}{(1-p)} \cdot \frac{\Gamma(\alpha+\beta)}{\Gamma(\alpha) \Gamma(\beta)} \cdot \frac{\left(y_{i}(p)-p\right)^{\alpha-1}}{(1-p)^{\alpha-1}} \cdot \frac{\left(1-y_{i}(p)\right)^{\beta-1}}{(1-p)^{\beta-1}} \\
\text { Let } \quad x_{i}=\frac{y_{i}(p)-p}{1-p},
\end{gathered}
$$

Then, with little algebraic manipulation, it can be shown that,

$$
\frac{1-y_{i}(p)}{1-p}=1-x_{i}
$$

Note that $d y_{i}(p)=(1-p) d x_{i}$ and that $x_{i}=0$ for $y_{i}(p)=p$. Therefore, Equation (10) can be rewritten as

$$
E(Y)=\int_{0}^{1} \frac{1}{1-p} \frac{\Gamma(\alpha+\beta)}{\Gamma(\alpha) \Gamma(\beta)}\left(p+x_{i}(1-p)\right) x_{i}^{\alpha-1}\left(1-x_{i}\right)^{\beta-1}(1-p) d x_{i}
$$

Now we can easily compute the expected value of the left-truncated beta distribution as follows;

$$
\begin{aligned}
E(Y) & =\int_{0}^{1} \frac{\Gamma(\alpha+\beta)}{\Gamma(\alpha) \Gamma(\beta)}\left(p+x_{i}(1-p)\right) x_{i}^{\alpha-1}\left(1-x_{i}\right)^{\beta-1} d x_{i} \\
& =p \int_{0}^{1} \frac{\Gamma(\alpha+\beta)}{\Gamma(\alpha) \Gamma(\beta)} x_{i}^{\alpha-1}\left(1-x_{i}\right)^{\beta-1} d x_{i} \\
& +(1-p) \int_{0}^{1} \frac{\Gamma(\alpha+\beta)}{\Gamma(\alpha) \Gamma(\beta)} x_{i}^{(\alpha+1)-1}\left(1-x_{i}\right)^{\beta-1} d x_{i} \\
& =p \int_{0}^{1} f\left(x_{i}\right) d x_{i}+(1-p) \int_{0}^{1} x_{i} f\left(x_{i}\right) d x_{i} \\
& =p+(1-p) \cdot \frac{\alpha}{\alpha+\beta} \\
& =p+\frac{\alpha(1-p)}{\alpha+\beta} \\
& =\frac{\alpha(1-p)+p(\alpha+\beta)}{\alpha+\beta} \\
& =\frac{\alpha-p \alpha+p \alpha+p \beta}{\alpha+\beta} \\
& =\frac{\alpha+p \beta}{\alpha+\beta}
\end{aligned}
$$

Note that $\frac{\Gamma(\alpha+\beta)}{\Gamma(\alpha) \Gamma(\beta)} x_{i}^{\alpha-1}\left(1-x_{i}\right)^{\beta-1}$ is a general beta distribution in the interval $(0,1)$ and hence its integral in the region $(0,1)$ is one. Also, it can be shown that,

$$
\sigma^{2}(Y)=\frac{(p-1)^{2} \alpha \beta}{(\alpha+\beta+1)(\alpha+\beta)^{2}}
$$

\subsection{Modeling Multidimensional Poverty Index Using the Beta Distribution}

Let $y_{i}(p)$ be a deprivation score as defined in Equation (2). Then $y_{i}(p)$ can be modeled using the left truncated beta distribution. In the subsequent proposition, we showed that the expected value of the left truncated beta distribution equals MPI computed using the Santos and Alkire (2011) method.

Proposition : Let $Y$ be a random variable taking values $y_{i} \in(0,1) ; i=1,2, \ldots \ldots \ldots \ldots, n$; denoting deprivation scores for individual $i$. Also let $p \in(0,1)$ be the deprivation threshold and $Y \sim \operatorname{Beta}(\alpha, \beta, p, 1)$; individual $i$ is deprived if $p \leq y_{i}<1$ and is given $y_{i}(p)$, otherwise an individual $i$ is non-deprived if $0<y_{i}<p$ and hence equate his/her deprivation score to 0 . Then it follows that MPI is the expected value of $Y$.

Proof.

From Equation (4) it follows

$$
A q=\sum_{i=1}^{n} y_{i}(p)
$$

On the other hand, we know that mean of the deprivation scores $[E(Y)]$ is computed as $\frac{\sum_{i=1}^{n} y_{i}(p)}{n}$. Thus, 


$$
\frac{\sum_{i=1}^{n} y_{i}(p)}{n}=\frac{A q}{n}
$$

Recall that $\mathrm{Y}$ is a left truncated beta distributed random variable and hence $E(Y)=\frac{\alpha+p \beta}{\alpha+\beta}$. Therefore,

$$
\begin{array}{ll}
E(Y) & =\frac{A q}{n} \\
\frac{\alpha+p \beta}{\alpha+\beta} & =\frac{A q}{n} \\
\frac{\alpha+p \beta}{\alpha+\beta} & =A \times \frac{q}{n} \\
A & =\frac{n}{q} \times \frac{\alpha+p \beta}{\alpha+\beta}
\end{array}
$$

Substituting Equation (3) and Equation (17) into Equation (5) as follows;

$$
\begin{aligned}
M P I & =H \times A \\
& =\frac{q}{n} \times \frac{n(\alpha+p \beta)}{q(\alpha+\beta)} \\
& =\frac{q}{n} \times \frac{n}{q} \times \frac{\alpha+p \beta}{\alpha+\beta} \\
& =\frac{\alpha+p \beta}{\alpha+\beta}
\end{aligned}
$$

\section{Results and Discussion}

\subsection{Multidimensional Poverty Index as Computed Using the Beta Distribution}

Table 3 presents the parameter estimates of beta distribution, MPI for Namibia computed using Equation (18), and its 95\% confidence interval. The parameters $\alpha$ and $\beta$ were estimated using the maximum likelihood method. The threshold value of 0.1172 was obtained by taking the threshold value of 0.33 under the Santos and Alkire (2011) method and then comparing the proportion of the deprivation under the entropy method and the Santos and Alkire (2011) method. A higher proportion of deprivation was observed when using the Santos and Alkire (2011) method compared to using the entropy method. One would expect a more or less similar proportion of deprivation when using both methods. Hence, to get a true picture of the deprivations, we adjusted the threshold value of 0.33 by setting the proportion of the deprived in the Santos and Alkire (2011) method to be equal to the proportion under the entropy method and we deduced the corresponding threshold, which was found to be 0.1172 .

Table 3. Namibia MPI using the beta distribution

\begin{tabular}{|c|c|c|c|}
\hline Alpha & Beta & Threshold $\mathrm{p}$ & MPI $(95 \%$ Confidence Interval $)$ \\
\hline 0.8721 & 2.6346 & 0.1172 & $0.4839(0.4837,0.4841)$ \\
\hline
\end{tabular}

The MPI in Table 3 shows that poor households experience about $48 \%$ of the deprivations as a share of the possible deprivations that would be experienced if all households were deprived in the three dimensions considered in this study (i.e. health, education, and living standards). Table 4 presents the Namibia regional MPIs computed using the beta distribution at a threshold of 0.1172 .

Table 4. Namibia regional multidimensional poverty index using the beta distribution

\begin{tabular}{|c|c|c|c|}
\hline Region & Alpha & Beta & MPI $(95 \%$ Confidence Interval $)$ \\
\hline Zambezi & 3.6718 & 4.1331 & $0.5325(0.5316,0.5334)$ \\
\hline //Karas & 1.5310 & 3.7991 & $0.3708(0.3698,0.3718)$ \\
\hline Erongo & 1.6753 & 5.1567 & $0.3337(0.3331,0.3343)$ \\
\hline Hardap & 1.4623 & 2.9606 & $0.4091(0.4078,0.4103)$ \\
\hline Kavango East & 1.9856 & 2.3221 & $0.5241(0.5226,0.5256)$ \\
\hline Kavango West & 4.3830 & 3.2455 & $0.6244(0.6235,0.6253)$ \\
\hline
\end{tabular}




\begin{tabular}{|c|l|l|l|}
\hline Khomas & 2.1683 & 5.5035 & $0.3667(0.3662,0.3672)$ \\
\hline Kunene & 2.3927 & 2.7339 & $0.5292(0.5280,0.5305)$ \\
\hline Ohangwena & 4.1441 & 3.6357 & $0.5874(0.5867,0.5882)$ \\
\hline Omaheke & 2.6872 & 3.2601 & $0.5161(0.5150,0.5172)$ \\
\hline Omusati & 4.5555 & 3.8982 & $0.5929(0.5923,0.5936)$ \\
\hline Oshana & 2.0191 & 3.2487 & $0.4556(0.4546,0.4565)$ \\
\hline Oshikoto & 3.0204 & 2.9459 & $0.5641(0.5632,0.5650)$ \\
\hline Otjozondjupa & 1.4645 & 2.7137 & $0.4266(0.4255,0.4278)$ \\
\hline
\end{tabular}

The results in Table 4 indicate that Kavango west is the worst multidimensionally poor region in the northern part of Namibia (MPI: $0.6244,95 \%$ CI: 0.6235 to 0.6253 ). Whereas, Oshana is the least multidimensionally poor region (MPI: $0.4556,95 \%$ CI: 0.4546 to 0.4565 ). In the central of Namibia, Omaheke (MPI: $0.5161,95 \%$ CI: 0.5150 to 0.5172 ) is the worst multidimensionally poor region, and Erongo (MPI: 0.3337, 95\% CI: 0.3331 to 0.3343) is the least multidimensionally poor region. The worst multidimensionally poor region in the southern part of Namibia is Hardap (MPI: $0.4091,95 \%$ CI: 0.4078 to 0.4103 ), and the least multidimensionally poor region is //Karas (MPI: $0.3708,95 \%$ CI: 0.3698 to 0.3718 ).

Overall, it can be observed that the worst multidimensionally poor region in Namibia is Kavango West (MPI: 0.6244, 95\% CI: 0.6235 to 0.6253 ), and the least multidimensionally poor region in Namibia is Erongo (MPI: 0.3337, 95\% CI: 0.3331 to 0.3343 ). This concurs with the findings of the NSA (2021), which pointed out that Kavango West was the poorest region in Namibia, with Erongo being the least poor region in terms of multidimensional poverty. Also, the findings of this study show that northern regions that include Kavango West, Omusati, Ohangwena, Oshikoto, Zambezi, and Kavango East are multidimensional poorest regions in Namibia. This result is in agreement with the findings of English (2016) indicating that poverty is more prevalent in the northern regions of Namibia.

\subsection{Sensitivity Analysis and Simulation of Beta Distribution}

A derivative-based sensitivity analysis was performed to assess the effect of changes in the parameters $\alpha, \beta$, and $p$ on the MPI. Firstly, to assess the effect of $\alpha$ on MPI, we took the partial derivative of Equation (18) with respect to $\alpha$ (i.e $\left.\frac{\partial M P I}{\partial \alpha}\right)$, which resulted to

$$
\frac{\beta(1-p)}{(\alpha+\beta)^{2}}, 0<p<1 \text { and } \alpha, \beta>0
$$

Equation (19) shows that MPI is an increasing function with respect to $\alpha$. That is, MPI increases with an increase in $\alpha$. Secondly, the effect of $\beta$ on MPI was assessed by taking the partial derivative of Equation (18) with respect to $\beta$, resulting in

$$
\frac{\partial M P I}{\partial \beta}=-\left[\frac{\alpha+p \alpha}{(\alpha+\beta)^{2}}\right], \quad 0<p<1 \quad \text { and } \quad \alpha, \beta>0
$$

Equation (20) reveals that MPI is a decreasing function with respect to $\beta$. This implies that MPI decreases with an increase in the $\beta$ value and vice versa.

Thirdly, the threshold value $p$ has a positive effect on MPI because the partial derivative of the MPI value with respect to $p$ (i.e. $\frac{\partial M P I}{\partial p}$ ) is a positive constant function of $\alpha$ and $\beta$.

$$
\frac{\beta}{\alpha+\beta}, \quad \alpha, \beta>0
$$

To further analyze the effect of the parameters $\alpha$ and $\beta$ on beta distribution function, we simulated the density function for different value combinations of $\alpha$ and $\beta$. 
(a)

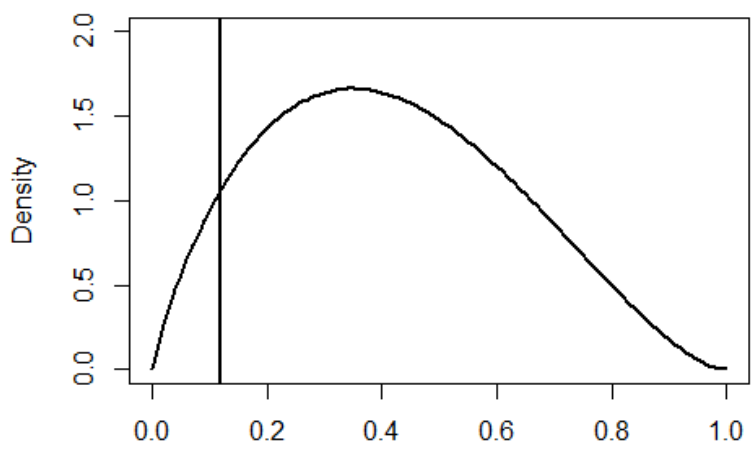

Deprivation scores (b)

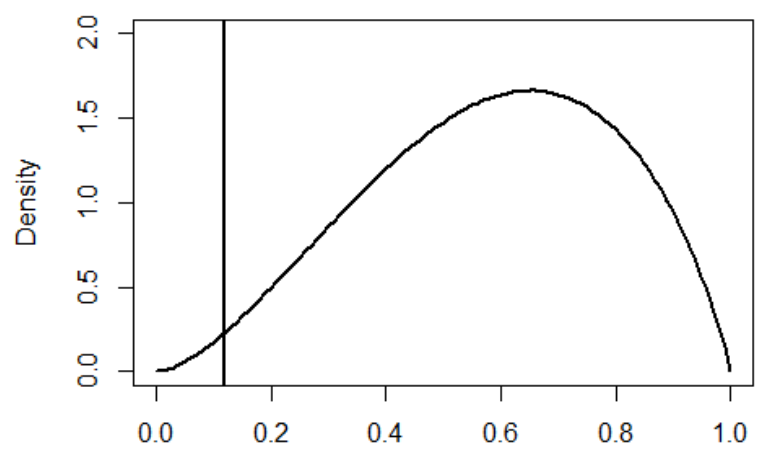

Deprivation scores

Figure 1. Beta distribution with a threshold of 0.1172

Figure 1 illustrates the behavior of beta distribution when the values of $\alpha$ and $\beta$ are switched. In this case, $\alpha=$ 1.8721 and $\beta=2.6346$ for Figure 1(a), and $\alpha=2.6346$ and $\beta=1.8721$ for Figure 1(b).

Figure 1(a) shows a slightly right-skewed distribution whereas Figure 1(b) is slightly left-skewed. This means that the shape and skewness of the distribution depend on the values of $\alpha$ and $\beta$. The mode of this distribution occurs at $y=(\alpha-1) /(\alpha+\beta-2)$ since $\alpha, \beta>1$.

(a)

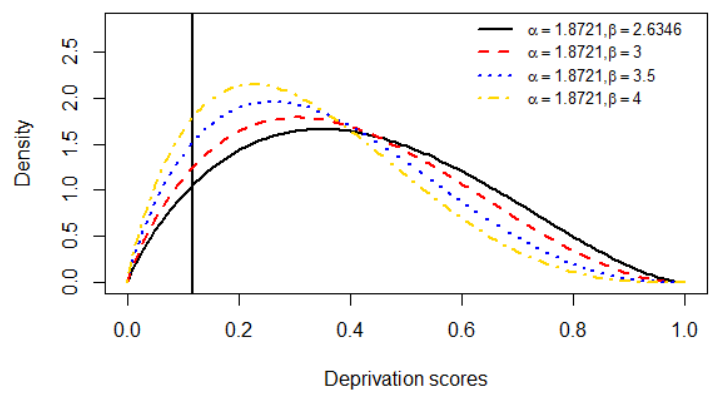

(c)

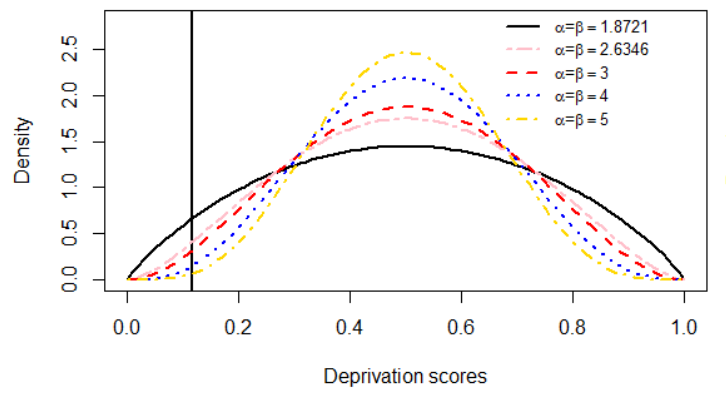

(b)

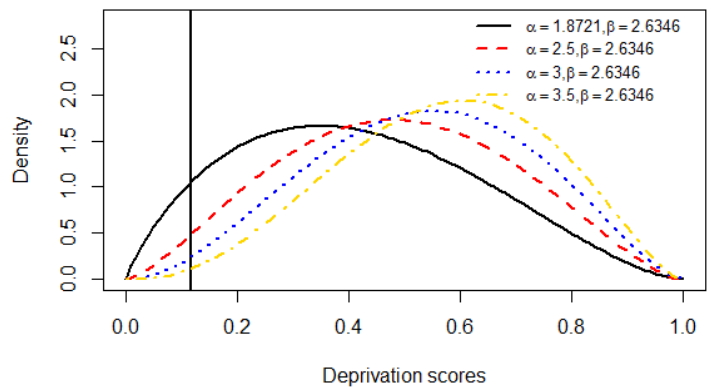

(d)

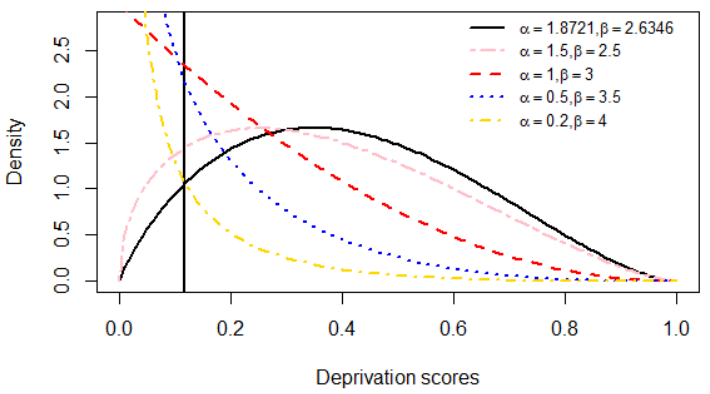

Figure 2. Beta distribution with different values of $\alpha$ and $\beta$

Figure 2(a) shows a beta distribution when keeping $\beta$ constant while increasing $\alpha$. Figure 2(b) illustrates a beta distribution for a constant $\alpha$ and increasing $\beta$. While Figure 2(c) portrays a beta distribution with equal and increasing $\alpha$ and $\beta$. Figure 2(d) shows a beta distribution for decreasing $\alpha$ and increasing $\beta$.

Increasing $\alpha$ and keeping $\beta$ constant shows that the distribution becomes strongly left-skewed as $\alpha$ increases (Figure 2(a)). On the other hand, by keeping $\alpha$ constant while increasing $\beta$, the distribution skewness switches to the 
right (Figure 2(b)). The beta distribution in Figure 2(c) is unimodal and symmetric about 0.5.

Increasing $\beta$ and decreasing $\alpha$ yields a reverse J-shaped beta distribution (Figure 2(d)). But, decreasing $\beta$ and increasing $\alpha$ yields a J-shaped beta distribution Figure 3.

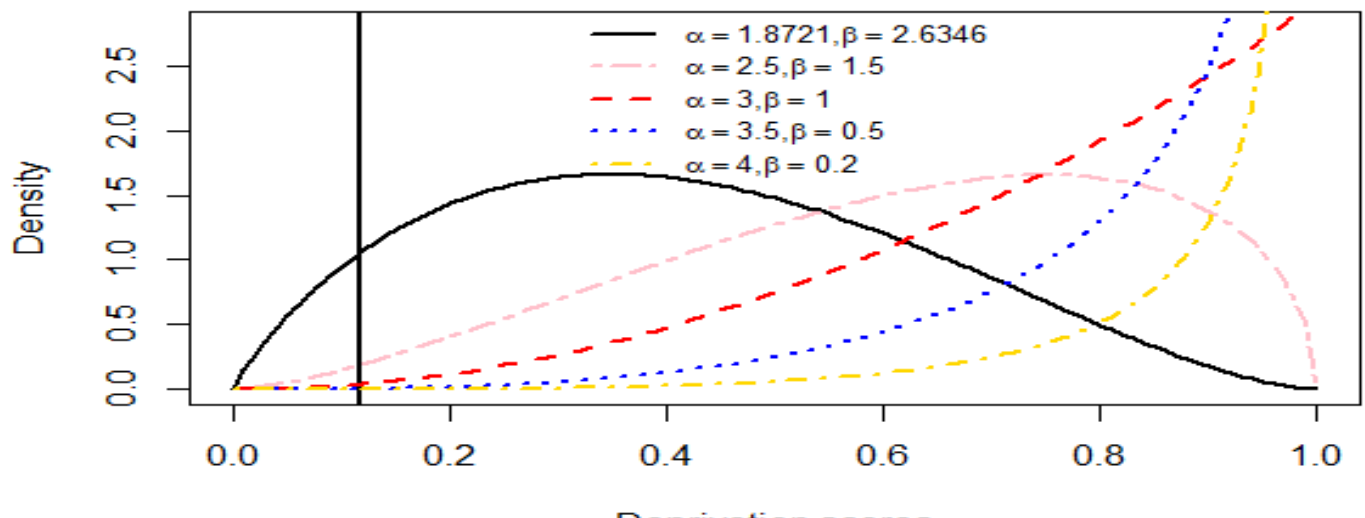

Deprivation scores

Figure 3. Beta distribution for decreasing $\beta$ and increasing $\alpha$

\section{Conclusion}

This study has shown that the beta distribution can be used to compute the multidimensional poverty index. To the researchers' knowledge, no other study has used beta distribution to model multidimensional poverty. Also, we have shown that beta distribution is useful in estimating MPIs at regional and national levels. Furthermore, it also allows quantifying the uncertainty around the computed MPI by specifying the variance (thus the confidence interval). The results from the study can also help identify areas that are highly affected by multidimensional poverty, and assist policymakers to formulate targeted poverty interventions intended to reduce poverty across the country. Furthermore, the study has revealed that the northern regions of Namibia are the poorest compared to the central and southern regions.

One of the limitations of this study is that the beta distribution does not provide the headcount ratio and the intensity of poverty. Another limitation of this study is that the multidimensional poverty index computed using beta distribution is highly dependent on the parameters $\alpha, \beta$ and $p$. This might have an impact on the computed MPI as the $\alpha$ and $\beta$ estimates are prone to some estimation errors.

\section{References}

Adepoju, A. (2018). Determinants of multidimensional poverty transitions among rural households in Nigeria (No. 2058-2018-5335).

Allen, R. C. (2017). Absolute poverty: When necessity displaces desire. American Economic Review, 107(12), 3690-3721. https://doi.org/10.1257/aer.20161080

Appiah-Kubi, K., Amanning-Ampomah, E., \& Ahortor, C. (2007). Multi-dimensional analysis of poverty in ghana using fuzzy sets theory. https://doi.org/10.2139/ssrn.3171635

Bellù, L. G., \& Liberati, P. (2005). Impacts of Policies on Poverty. Absolute Poverty Lines.

Betti, G., D’Agostino, A., \& Neri, L. (2002). Panel regression models for measuring multidimensional poverty dynamics. Statistical methods and applications, 11(3), 359-369. https://doi.org/10.1007/BF02509832

Ciani, M., Gagliardi, F., Riccarelli, S., \& Betti, G. (2019). Fuzzy measures of multidimensional poverty in the Mediterranean area: A focus on financial dimension. Sustainability, 11(1), 143. https://doi.org/10.3390/su11010143

De, L. (2017). Poverty and its measurement. Instituto Nacional De Estadistica, 1-34.

Decerf, B. (2017). Conciliating Absolute and Relative Poverty: Income Poverty Measurement Beyond Senâ s Model (No. 1701).

English, J. (2016). Inequality and Poverty in Namibia: A Gaping Wealth Gap. Clint Borgen https://borgenproject.org/wpcontent/ uploads/The_Borgen_Project_Logo_small.jpg.

Gao, S., \& Sun, K. (2020). Poverty measure based on hesitant fuzzy decision algorithm under social network media. 
Symmetry, 12(3), 384. https://doi.org/10.3390/sym12030384

Jolliffe, D., \& Prydz, E. B. (2016). Estimating international poverty lines from comparable national thresholds. The Journal of Economic Inequality, 14(2), 185-198. https://doi.org/10.1007/s10888-016-9327-5

Marx, I., \& Van Den Bosch, K. (2007). How poverty differs from inequality. On poverty measurement in an enlarged EU context: conventional and alternative approaches.

Mellish, J. (2016). Relative Poverty-a Measure of Inequality, not Poverty.

Mothkoor, V., \& Badgaiyan, N. (2021). Estimates of multidimensional poverty for India using NSSO-71 and-75 (No. 2021/1). WIDER Working Paper. https://doi.org/10.35188/UNU-WIDER/2021/935-8

Mukui, J. W. (2017). Construction of multidimensional poverty index of Kenya using the Alkire-Foster method.

Nam, S. J. (2020). Multidimensional poverty among female householders in Korea: Application of a latent class model. Sustainability, 12(2), 701. https://doi.org/10.3390/su12020701

Namibia Statistics Agency. (2021). Namibia multidimensional poverty index (MPI) report 2021.

National Planning Commission. (2015). Namibia Index of Multiple Deprivation.

Oktavianti, E., Komala, N., \& Nugrahani, F. (2019, April). Simple multi attribute rating technique (SMART) method on employee promotions. In Journal of Physics: Conference Series (Vol. 1193, No. 1, p. 012028). IOP Publishing. https://doi.org/10.1088/1742-6596/1193/1/012028

Pochun, M. (2002). Measures of poverty and inequality in developing countries.

Santos, M. E., \& Alkire, S. (2011). Training material for producing national human development reports. MPI: Construction and analysis. Oxford: Oxford Poverty and Human Development Initiative.

Walker, R. (2019). Multidimensional poverty: Whose poverty is it?. In Routledge International Handbook of Poverty (pp. 33-48). Routledge. https://doi.org/10.4324/9780429058103-4

Walker, R., Tomlinson, M., \& Williams, G. (2008). Multi-dimensional measurement of poverty and wellbeing: A UK case study.

Wang, S. S., Huang, T. Y., Chen, H., Liu, M. Y., \& Xue, H. L. (2018). Application of fuzzy comprehensive evaluation model based CRITIC weighting in water quality evaluation. Water Resources and Power, 06.

Zaninetti, L. (2013). The initial mass function modeled by a left truncated beta distribution. The Astrophysical Journal, 765(2), 128. https://doi.org/10.1088/0004-637X/765/2/128

\section{Copyrights}

Copyright for this article is retained by the author(s), with first publication rights granted to the journal.

This is an open-access article distributed under the terms and conditions of the Creative Commons Attribution license (http://creativecommons.org/licenses/by/4.0/). 